clear away offensive secretions and lessen discomfort. In such cases the frequent application of hot poultices was of great service. In restlessness and sustained pyrexia cold and tepid spongings were useful to promote sleep, and sulphonal in some instances proved a valuable bypnotic. The cases were treated in bright and well-ventilated wards, maintained at a temperature of $56^{\circ}$ to $60^{\circ} \mathrm{F}$, the average cubic space per bed being about $2000 \mathrm{ft}$. The diet during the pyrexial stage consisted of milk, beef-tea, egge, and ice, after which a more solid diet of milk pudding, bread. and-butter, with fish or meat, was given, and at the same time baths were ordered on alternate days. Stimulants were only cmployed in severe cases, usually in the form of brandy or champagne. Uncomplicated cases were allowed to get up at the end of the third week, and, except in wet weather, sent out of doors for several hours usually each day, due care being taken that flannel was worn next the skin and the clothing otherwise warm and generally sufficient. Complications were dealt with as they arose, and, with the exception of otorrhoe, were rarely seen after the third week.

With reference to the treatment of scarlatinal nephritis, I am not in the habit of withholding eggs as an article of diet when the secretion of urine has become fairly established, especially if there be much loss of albumen and consequent anæmia. Although the employment of eggs would be condemned by many on theoretical grounds, I can only say that I have followed the practice for several years with the best resulte. Though milk should be the staple food, patients will more rapidly put on flesh, lose their albumen, and gain colour if not withheld from a light solid diet, including boiled eggs. With the exception of perchloride of iron and the free use of purgatives, I am not in the habit of using drugs in ordinary cases. A death from scarlatinal nephritis is an event of the greatest rarity. In this series of over 1000 cases of scarlatina only one death occurred from that cause, the child being admitted with nephritis, and dying a few days after in convulsions.

\section{A PROBLEM IN THE ELECTRICAL REACTIONS OF MUSCLES.}

BY J A M E CA G NEY, M.A., M.D., ELECTRO-THERAPEUTICAL OFFICER, ST. MARY'S HOSPITAY; PHYSICIAN,
HOSPITAL FOR EPILEPSY AND PARALYSIS, REGEN'S PARK.

WHATEVER controversy there may be as to the usefulness of electricity in the treatment of nervous diseases, there can be no doubt that it has a considerable sphere in diagnosis, and from that point of view is well worthy of study. The emancipation of medical electricity from degraded uses and its dedication to the purposes of science may be said to date from the establishment as a proximate and partly understo od truth of the broad principle underlying what is known as the reaction of degeneration. This prineiple, however, by no means covers all observable facts. In the scientific order facts precede theories, and they may have a practical value of their own independently of their explanation. I I desire to call the attention of others to an appearance which has engaged mine for some years, and to which, rightly or wrongly, I have come to atcach some practical significance. There is a phenomenon which is famliar to everyone who employs electric currents for the treatment of paralysis, and whose opportunity and resource supply him with sufficient variety of material. To take as an example a case of paralysis from lead poisoning. When a fairly strong faradaic current is applied to the extensors in the forearm, other muscles, usually including the more accessible of the flexors, respond, and with a vigour proportionate to the intensity of the current. This may be shown in almost any case where the anatomical distribution of the paralysis enables one to cause an efficient stimulation of more distant muscles while those beneath the exciting electrode remain inert. It is usually said that the current is " conducted through." The fact, of course, is significant and invariably watched for. I have seen a distinguished physician point to it as if it indicated a more than usually desperate state of things. In point of fact, I believe it can always be elicited where the reaction of degeneration exists if there are healthy muscles near, and that it indicates nothing but a certain strength of current-an indication more satisfactorily provided by measuring instruments.

There are cases, however, which present an appearance very similar to this, invariably, I believe, confounded with it, and yet, if I mistake not, essentially distinct from it. I will take for illustration another case. A boy had been sent to me to be treated for infantile paralysis. I will speak of the disease as such because it had been so named by three eminent surgeons who had it under observation at an earlier period than I. Moreover, it would be difficult to assign the case to any other of the recognised clinical groups. At the same time there weze in its history and in other particulars certain things to arrest. the attention, if not to suspend the judgment. The boy had broken his arm about the lower epiphysis of the humerus, and it remained deformed. When the splint was removed he was found to be paralysed. I saw him between two and three months afterwards. The pectorals, trapezius, sternomastoid, and scapular muscles were much wasted. Of the deltoid there was scarcely any substance left, and the head of the humerus was widely separated from its cavity. The vertebral border of the scapula was tilted backwards, probably from paralysis of the serratus magnus. The rhomboids wers affected. It was impossible to raise the point of the shoulder or the arm, or to draw the arm across the front of the body, and to flex or supinate the forearm. Extension of the wrist was verv feeble. The electrical reactions were remarkable. In the first place, they varied in the same part at short in. tervals, almost from day to day. This was particularly so in the rhomboids and the deltoid. When the latter muscle was stimulated with a galvanic current it was found that parts of it which one day gave no response contracted readily the next, and distinct portions would for a time assume a healthy action, though a short time before they had given an evident reaction of degeneration, and the next day perhaps would do so again. The inner head of the triceps was somewhat atrophied and inert to all stimuli ; the outer and long heads reacted normally, showed increased excitability, and were in a state of feeble tonic contraction. The supinator longus was directly excitable. When with a faradaic eurrent the exciting electrode was placed over the supinator longus that muscle contracted, but the triceps did so much more energetically. If the electrode was applied, not over the supinator longus but over the extensors in the forearm, the same thing occurred, and the force of contraction in the triceps seemed to vary with the distance of the electrode from a point about the middle of the supinator longus. When the triceps contracted the anconeus did so too. Now, there are some facts which serve to distinguish this contraction in the triceps and anconeus from that which occurs in the flexors of the wrist when a current is said to be "conducted through," as in lead palsy. 1. A very weaks current sufficed to produce it-such a current as would cause no contraction in the apparently healthy supinator. 2. The effect on the triceps was appreciably delayed, and when both the supinator longus and the triceps were made to contract the former did so first. 3. No such effect as that. on the triceps occurred in other muscles much nearer thas. it to the exciting electrode. It ocsurred to one of courso that the over-excitability of the triceps and its spastic state might account for the events. This is not ny belief, and would offer the following reasons: 1 . The stimulus applied to the supinator longus was more efficient than that applied directly to the triceps. 2. The same effect was simultaneously produced in the extensors of the wrist, which directly were hardly excitable at all. In other words the motor point for the entire group-trieeps, anconeus, extensors, and supinator longus-was situated in the latter. 3 I have frequently observed this "reflected" stimulus in other cases, and I have not found it asso. ciated with spasms or increased exeitability. The: stimulus is not simply conducted through the tissues. It is necessary to seek for some special cause determining its path. In doing so one is, of course, struck by the fact that the whole train of events is concerned with the distribution of a single nerve, the museulo-spiral. I may at once mention that in every case which has come under my notice I have observed a similar and more or less immediate anatomical relation as to nerve-supply between the muscles illustrating a "reflected" stimulus. I adopt this word "reflected" for brevity's sake, and I do so with deference to the physiologists, having a desir to imply 
theory, as I have no intention of formulating one. I might suggest, indeed, that there is between this event and those which are known in physiology as the "paradoxical contraction" and "secondary tetanus from a nerve" (Landois and Stirling, third edition, p. 562) a certain analogy. We hear a great deal of functional diseases of the nervous system, but nothing, so far as I know, of functional disorder of a nerve fibre. We know that a nerve fibre can conduct impressions in either direction; we assume that as a fact it does so in one only. It is thought that the guiding influence resides in the end organ, and may be said to be its function. Dr. Waller, in the very admirable paper recently submitted to the Neurological Society, has shown that the motorial end plate may manifest fatigue, while the remaining elements in the neuro-muscular chain keep their energy unimpaired. If the motorial end plate, when fatigued, can temporarily block the path of volitional impulses, it may do this permanently under other circumstances. In view of such a possibility, it is well to keep an open mind, and not to assume too hastily that in the case of the nerve fibre there is no condition intermediate between absolute integrity on the one hand, and, on the other, total destruction, either actual or impending, -a destruction in which the terminal muscle fibre will certainly be involved. The peculiar reaction which I have described seems to prove that in a given nerve, or part of it, the cause which limits the effect of an induction shock to the muscle at its peripheral extremity may be suspended. Observation will show that under these circumstances the muscle may retain its function for many months while its electrical reactions are perverted and voluntary power is lost, and this condition may, and does often, occur side by side with degenerations due to organic disease. It is most apt to take place in neurotic subjects. I have seen it in crutch paralysis and in paresis of the upper extremity from pressure of a splint. In a case of spinal hæmorrhage sent to me by Dr. Broadbent, where uaralysis of the sphincters and of certain muscles of the leg persisted, and probably still persists, the reaction was ob. tainable during two years, and disappeared gradually as the muscles manifesting it regained something of bulk and strength. It may be met with most often in infantile paralysis, and there, as elsewhere, it is pro tanto a favourable indication for treatment. It seems to me to represent a large part of the recoverable element in that disease, and so long as it persists there is ground for abundant hope that the judicious use of galvanism and other measures will be productive of benefit, though not of course of cure.

Welbeck-street, $\mathbf{w}$.

\section{THE CURE OF "INTERNAL DERANGEMENT" OF THE KNEE-JOINT BY MANUAL OPERATION}

By NOBLE SMITH, F.R C.S. EDIN., L R C.P. LoND., SURGEON TO THE ALL SAINTS CHILDREN'S HOSPITAL.

A CENTURY has passed since that eminent surgeon, William Hey, of Leeds, drew attention to an "Internal Derangement of the Knee-joint," for which he devised a remedy by manual operation. Another Leeds surgeon, Samuel Smith, following upon the same lines of observation, also found many similar cases, and succeeded in obtaining the same curative results by the same remedial method. After William Hey, besides Samuel Smith, we find Samuel Hey, grandson of William Hey, Sir Astley Cooper, Syme, Sir Benjamin Brodie, and others publishing similar cases treated successfally by Hey's method. In the present age of brilliant and successful operative surgery these facts are sometimes forgotten, and it has even been suggested that a cutting operation which involves the opening of the joint is the only means of relieving the disabilities which accompany this troublesome and often severely painful affection. That opening of the joint may be justifiable in some exceptional cases may certainly be conceded, but it has been abundantly proved that a large majority of the patients who suffer from these "derangements" can be absolutely and permanently cured by manual operations alone.

Into the question of the exact nature of the abnormal conditions which pertain it is not, I think, necessary here to enter. Several abnormal conditions have been shown to exist in cases which have been subjected to the knife, either during life or after death; suffice it here to state that displacement of one or other of the semilunar fibro-cartilages, generally the internal one, seems commonly to have been the chief or only derangement in the cases which have answered the description first given to the accident by Hey. Eight years ago I published a series of cases which had come under my care for derangement of the knee-joint, in all of which Hey's plan of manipulation had perfectly suc ceeded. From time to time since then similar cases have come before me, the histories of which go to confirm the statements of the older writers and my former experiences. The question arises as to whether this manual reduc. tion is permanent in its effects, or whether a tendency to relapse does not render the more severe operative procedure justifiable and desirable. Hey refers to one case of relapse in four cases recorded, the accident recurring two years after reduction, but it was very easily put right. Samuel Smith states that he had been surgeon to the Leeds Infirmary for thirty-two years, and he thought he had rarely passed a year without seeing a case, and he was sure that he had seen as many as four or five cases in a year. He refers to Hey's mode of "cure," but he also describes some relapses. One young lady stated that she had suffered from this derangement at least six times in four or five years, and on each occasion (excepting the first) Mr. Samuel Smith had been at hand to give her relief.

After consideration of these cases of relapse I thought that the displaced cartilage probably became changed in form from pressure in its unnatural position, and that, although reduced, it would have a tendency to work its way out again unless retained in its normal place sufficiently long for it to regain its natural shape. Acting upon this theory, I insisted upon the importance not only of reducing the displacement in the first instance, but of repeating the manipulation daily for a few days, and then once every two or three days for a fortnight or more, according to circumstances, with a view to keeping up the good position until the parts had quite recovered their normal condition. This plan has proved remarkably successful, and I have practically met with no relapses after adopting this treatment in cases where $I$ have once succeeded in effecting a reduc. tion. I am now able to give the subsequent history of the cases which I recorded eight years ago, as well as to refer to other instances of permanent cure.

CASE 1.-Mrs. C. M-. The displacement first occurred at the age of fifty-eight, in the year 1880 . There were many relapses during the first six months, each of which was reduced at the time, but treatment was not followed up as above described. The displacement recurred a great many times during the first few weeks, but the tendency gradually lessened, so that during the second six months it only occurred once. From that time up to the present (August. 1890), nine years, no relapse has taken place.

CASE 2-Miss W- aged serenteen, seen in August, 1881 (three years after the first acciden!). The displace ment had never been thoroughly reduced until this time. I followed up the passive movements for nearly three weeks, for the first fortnight daily. The result was excellent, and two years afterwards the patient wrote to me from Boston, U.S.A., informing me that she was perfectly well and could dance and use the leg without the least difficulty. The knee had remained sound since the treatment by com. plete reduction, whereas during the three previous years she had been continuously lame.

CASE 3-Mr. H-, aged thirty, came to me in 1881 . He had been crippled by his bad knee continuously for four years, and occasionally for the preceding six years. He had not been able to place the leg in a straight position for the five years before I saw him. It took half an hour so reduce the displacement, but when accomplished the patient felt perfectly sound. I repeated my manipulations at intervals of a few days for about three weeks, and when 1 saw him about a year ago he had remained quite well the whole time, a period of eight years.

I have treated many cases since then, all with good results. I will describe the most severe case.

Miss $\mathrm{H}-$, aged forty, consulted me on Nov. 11 th, 1882 . She first injured her knee by a fall in the Alps in 1867 . She had to be carried down the glacier to Chamouni. She suffered extreme pain, but this was suddenly relieved during her transit by a second fall, caused by her carriers slipping and upsetting her. This seems to show the nature of the 